\title{
Editorial Comment: Deciphering the "Metastasome" Leads to Novel Hypotheses on Understanding the Evolution of Metastasis and Implicates Consequences for Metastasis Therapy and Prevention
}

\author{
Heike Allgayer ${ }^{1,2}$
}

Published online: 27 February 2019

(C) Springer Nature B.V. 2019

The utmost challenge in cancer therapy undoubtedly still is the successful combat or even prevention of metastasis, which still can arise even years after successful primary tumor therapy or already during an ongoing, even personalized therapy of a cancer patient. Still, metastases are by far the most frequent causes for cancer deaths and we still are facing a situation of rather fragmented research at metastasis, a still incomplete understanding of metastasis evolution, and clinical protocols which often are not, or cannot, be specifically designed to target the metastatically relevant tumor cell clones.

Different hypotheses have been put forward so far on the genesis of metastasis (e.g., [1, 2]), reaching from a common clonal origin between a primary tumor and metastatic lesions (paralleling discussions around the cancer stem cell hypothesis), over early- and late stage dissemination models, up to hypotheses of a completely independent genesis and evolution of a primary tumor and metastatic lesion.

In a recent study [3] on the Whole Genomes of colorectal cancer metastases (the "metastasome") which were compared with the corresponding primary colorectal cancers of the same patients, subtracting the corresponding normal background tissues, we think we can suggest some answers to such questions on metastasis evolution and add, or support, particular hypotheses:

In this study [3], we show evidence suggesting that the primary tumor and the metastasis originate from a common ancestor clone indeed, and then undergo truncal separation. We hope that our findings in this context will be of interest not

Heike Allgayer

heike.allgayer@medma.uni-heidelberg.de

1 Department of Experimental Surgery-Cancer Metastasis, Medical Faculty Mannheim, Ruprecht Karls University, Ludolf Krehl Str.13-17, 68167 Mannheim, Germany

2 Centre for Biomedicine and Medical Technology Mannheim (CBTM), Mannheim, Germany only for the metastasis research but also the cancer stem cell research community. An average of about $65 \%$ of somatic mutations, for example, were shared between the primary tumor and corresponding metastasis, and mutational processes ongoing within particular mutational signatures clearly appear more truncal, whereas others appear more private to primary tumors or metastases after truncal separation. It remains up to further investigations whether the genomic lesions found to be common to both primary and metastasis would describe what we assumed up to now to be a "cancer stem cell", or whether it rather would be these together with the specific changes seen additionally in the metastasis. We furthermore show that the metastasis, as compared to the corresponding primary, has a higher mutational rate and shows a specific initiation of genetic programs important for site specific colonization. This of course is interesting especially in the context of the highly timely field of the lively crosstalk between tumor cells and diverse microenvironments, a systemic priming of metastatic niches, and diverse hypotheses on a molecular imprinting, vice versa, of the microenvironment and the tumor cells. In our publication which primarily studies metastases to the liver of colorectal cancers, we found that mutations specific to the metastasis are enriched in mutations concerning molecules within the PI3K-Akt signaling network, cell adhesion programs, extracellular matrix remodeling, but especially also hepatic stellate cell activation programs which would speculate a specific priming for metastasis formation in the liver. This clearly supports the hypothesis that genetic programs important for site-specific colonization to a particular organ site are initiated in metastasizing cancer cells, either in the process of a systemic or microenvironmental interaction of the cancer cells, or during further clonal differentiation and selection within the metastatic process.

Moreover, we confirm the Vogelstein sequence for colorectal cancer progression [4] but at the same time add some novel elements which, as we hope, support a deeper understanding of metastasis evolution and the progression of colon cancer. 
Again, in the context of tumor cell/microenvironment interactions, previously undescribed mutations, e.g., within CBL and CBLB 3'UTR regions, might be interesting for getting deeper insights into immunomodulatory actions of tumor cells during cancer progression and metastasis. Furthermore, besides other examples, we found mutations within the guanine nucleotide exchange factors $A R H G E F 33$ and $A R H G E F 7$, and it remains to be investigated whether, since we found them to be mutually exclusive with $R A S$ mutations, might have a similar function to activating $K-R A S / N-R A S$ mutations within EGFRinitiated signaling and EGFR-based therapy. This and further findings might suggest specific clinical consequences. For example, we show that metastases have specific genomic changes (19\% of all small somatic mutations detected), some of which being novel mutations within already targetable molecules. These were not detected in whole genome sequencing of the corresponding primary tumors. This means that even in current strategies of modern Comprehensive Cancer Centers (CCCs) which match primary and first line, even personalized, therapy to the genome of the individual primary tumor, there are still considerable chances to therapeutically miss the metastatically relevant tumor cell clones. This might be one highly likely explanation for the unchanged situation that metastases still arise after concerted primary tumor therapy and still lead to about $90 \%$ of cancer-related deaths.

Therefore, we sincerely hope that our data, besides stimulating more detailed hypotheses about colon cancer progression, metastasis evolution, cancer stem cells and metastasisinitiating cells, can invite clinical opinion leaders in oncology and related fields to take our findings into ongoing interdisciplinary considerations of refining personalized therapy concepts, so that they might open new chances to truly combat, or in long-term even prevent, metastasis.

Acknowledgements HA was supported by the Alfried Krupp von Bohlen und Halbach Foundation, Essen, the Deutsche Krebshilfe, Bonn (70112168), the Deutsche Forschungsgemeinschaft (DFG, grant number AL 465/9-1), the HEiKA Initiative (Karlsruhe Institute of Technology/ University of Heidelberg collaborative effort), Dr. Hella-BuehlerFoundation, Heidelberg, the DKFZ-MOST Cooperation, Heidelberg (grant number CA149), and the DKFZ-Heidelberg Center for Personalized Oncology (DKFZ-HIPO)-Initiative (H032 and H027).

Publisher's Note Springer Nature remains neutral with regard to jurisdictional claims in published maps and institutional affiliations.

\section{References}

1. Valastyan S, Weinberg RA (2011) Tumor metastasis: molecular insights and evolving paradigms. Cell 147:275-292

2. Massague J, Obenauf AC (2016) Metastatic colonization by circulating tumour cells. Nature 529:298-306

3. Ishaque N, Abba ML, Hauser C, Patil N, Paramasivam N, Huebschmann D, Leupold JH, Balasubramanian GP, Kleinheinz K, Toprak UH, Hutter B, Benner A, Shavinskaya A, Zhou C, Gu Z, Kerssemakers J, Marx A, Moniuszko M, Kozlowski M, Reszec J, Niklinski J, Eils J, Schlesner M, Eils R, Brors B, Allgayer H (2018, Nov 14) Whole genome sequencing reveals novel hypotheses on metastasis evolution and therapy in colorectal cancer. Nat Commun 9(1):4782. https://doi.org/10.1038/s41467-018-07041-z

4. Vogelstein B, Kinzler KW (1993) The multistep nature of cancer. Trends Genet $T I G$ 9:138-141 